\title{
Whole-Genome Sequencing of Pigeonpea: Requirement, Background History, Current Status and Future Prospects for Crop Improvement
}

\author{
Rachit K. Saxena and Rajeev K. Varshney
}

\begin{abstract}
Despite of being a very important crop, pigeonpea did not have genomic resources until 2005. Pigeonpea Genomics Initiative (PGI) supported by Indian Council of Agricultural Research (ICAR) under Indo-US Agriculture Knowledge Initiative was the first major initiative that delivered first set of molecular markers in large numbers, first set of mapping populations, first set of transcriptome assemblies, etc. Subsequently, two consortia-1) International Initiative for Pigeonpea Genomics (IIPG), led by International Crops Research Institute for the Semi-Arid Tropics (ICRISAT) and 2) Led by National Research Centre on Plant Biotechnology (NRCPB) - delivered two draft genome assemblies for Asha (ICPL 87119) variety. In summary, all these genomic resources transformed pigeonpea from an 'orphan crop' to 'genomics resources-rich crop'. After publication of draft genome sequences, a detailed plan was developed to utilize draft genome information for pigeonpea improvement. This plan in the form of a proposal was approved by Ministry of Agriculture, Government of India and United States Agency for International Development (USAID) - India. In addition to this major project, two additional projects were funded by Department of Biotechnology, Government of India. All these efforts have established high-density genotyping platforms such as genotyping by sequencing (GBS) and 'Axiom ${ }^{\circledR}$ CajanusSNP Array', produced the first generation HapMap, generated whole-genome re-sequencing data of $>400$ pigeonpea lines, evaluated several mapping populations for desired traits, established marker-trait association for several traits of interest to breeders and also identified best-performing lines. Additionally, multi-parent advance generation inter-cross (MAGIC) and nested association mapping
\end{abstract}

R.K. Saxena $(\bowtie) \cdot$ R.K. Varshney

International Crops Research Institute for the

Semi-Arid Tropics (ICRISAT), Patancheru 502324,

India

e-mail: r.saxena@cgiar.org 
(NAM) populations are being developed. With the availability of above-mentioned information, next few years will be witnessing application of genomics-assisted breeding for pigeonpea improvement. It is anticipated that improved pigeonpea lines developed through genomics interventions will reach to farmers' fields and elevate the game towards pulse sufficiency for poor farmers in arid and semi-arid regions of the world in near future.

\subsection{Introduction}

Much has been written on the importance of pigeonpea in previous chapters of this book and in many other published articles. However, until recently very few breeding programs have remained engaged in systematic pigeonpea research involving modern biology approaches. Moreover, the genetic material used in these breeding programs have limited genetic base. There is a need to diversify the genetic base and deploy modern approaches such as genomics-assisted breeding (GAB) for pigeonpea improvement. GAB has helped development of superior varieties and hybrids in several crops, in cereals (Varshney et al. 2005, 2006, 2010a) and legumes such as chickpea (Varshney et al. 2013) and groundnut (Varshney et al. 2014). The pigeonpea crop, however, remained untouched by the genomics revolution until 2005 and resulted as an 'orphan crop legume'. To enable GAB in this crop, the Pigeonpea Genomics Initiative (PGI) was started in late 2006 with financial support from Indian Council of Agricultural Research (ICAR) under the umbrella of Indo-US Agricultural Knowledge Initiative (AKI), US National Science Foundation's Plant Genome Research Program and the Generation Challenge Program (GCP). As a result of intensive efforts in PGI and several other programmes, a significant amount of genomic resources such as molecular markers, mapping populations and genetic maps was developed in pigeonpea (Varshney et al. 2010b). These collaborative efforts significantly benefited pigeonpea research community and transformed an 'orphan crop legume' to 'genomic resources-rich crop'. Although thousands of molecular markers and a number of mapping populations were developed in pigeonpea under PGI, very few genetic maps with low level of marker density and only three quantitative trait loci (QTL)-based studies were conducted (Bohra et al. 2012; Gnanesh et al. 2011; Kumawat et al. 2012). This has happened mainly because of low level of genetic diversity present in the pigeonpea-cultivated gene pool. To overcome the low level of genetic diversity bottleneck in the cultivated gene pool and for deploying GAB, following two options were considered: (1) develop novel genetic material with enhanced genetic diversity and (2) systematically scan entire pigeonpea genome for all possible variations. In PGI, initially a clone-by-clone approach was proposed to sequence the pigeonpea genome. However, due to lack of funds through PGI and availability of low-cost and high-throughput next generation sequencing (NGS) technologies encouraged pigeonpea genomics community for developing genome sequence based on whole-genome shotgun approach. At the later stage, International Initiative for Pigeonpea Genomics (IIPG) (http:// ceg.icrisat.org/gt-bt/iipg/Home.html) was floated to sequence genome and developed other genomic resources. As a result of efforts from IIPG and National Research Centre on Plant Biotechnology (NRCPB), two drafts of pigeonpea genome were published in Nature Biotechnology (Varshney et al. 2012) and Journal of Plant Biochemistry and Biotechnology (Singh et al. 2012). Nevertheless, the genomic resources developed in PGI were pivotal in both pigeonpea genome sequencing projects. 


\subsection{Genome Sequence Initiatives}

The year 2012 can be consider a significant year not only for pigeonpea but for the legume community. As mentioned above, IIPG and NRCPB delivered draft pigeonpea genome sequence almost at the same time, one was based on Illumina sequence data (Varshney et al. 2012) and the other one was based on 454 GS-FLX sequence data (Singh et al. 2012). These efforts delivered the genome sequence information of the first grain legume as well as the second food legume, after soybean to help increase the efficiency of pigeonpea improvement by integrating biotechnological tools in conventional breeding and to utilize the genome information of pigeonpea for analysing other legume species. It was also anticipated that the pigeonpea genome information will be useful to understand the genetic basis of hybrid vigour in pigeonpea, develop new methods for hybrid breeding.

Background history and glimpse of the two pigeonpea genome initiatives have been presented below:

\subsubsection{Draft Genome Sequence from IIPG (Varshney et al. 2012)}

IIPG delivered the genome sequence through partnership of 30 scientists from 12 institutes in 6 countries and published in Nature Biotechnology. Initial partners in the initiative were International Crops Research Institute for the Semi-Arid Tropics (ICRISAT), Patancheru, India; CGIAR Generation Challenge Programme (GCP), Mexico; Beijing Genomics Institute (BGI)-Shenzhen, Shenzhen, China; University of Georgia, Athens, Georgia, USA; National Center for Genome Resources (NCGR), Santa Fe, New Mexico, USA; University of North Carolina, Charlotte, North Carolina, USA; National University of Ireland Galway (NUIG), Botany and Plant Science, Galway, Ireland; University of
California, Davis, California, USA; Monsanto Company, Creve Coeur, Missouri, USA; Cold Spring Harbor Laboratory, Cold Spring Harbor, New York, USA; Department of Biology, University of Copenhagen, Denmark; BGI-Americas, Cambridge, Massachusetts, USA. This work received partial funding and in-kind support from CGIAR, GCP, US National Science Foundation, BGI-Shenzhen, China and ICRISAT, India. The decoding of pigeonpea genome was also backed by Indian Council of Agricultural Research (ICAR), India and supported through financial support to some earlier work that was used for analysing genome sequence data.

It is important to mention that above-mentioned partnership was accomplished without any specific funded project for the purpose. The two most important features of this partnership includes following: (1) total expenditure of $<\mathrm{US} \$ 200 \mathrm{~K}$, whereas in general genome sequencing projects involve millions of US\$s, (2) completion of pigeonpea genome sequencing in just about 2 years, unlike many other previous genome sequencing projects (of the same or even smaller genome sizes) generally took 5-10 years or even more (http://www.icrisat.org/newsroom/newsreleases/icrisat-pr-2011-media20.htm).

The consortium used 'ICPL 87119', popularly known as 'Asha' a pigeonpea variety for the generation and analysis of genome sequence. Illumina sequencing technology was used to generate $237.2 \mathrm{~Gb}$ of sequence data from 22 different insert size libraries ranging from $180 \mathrm{bp}$ to $20 \mathrm{~Kb}$. This sequence data along with Sanger-based BAC-end sequences and a genetic map assembled $\sim 73 \%(605.78 \mathrm{Mb})$ of the 833-Mbp pigeonpea genome. Genome analysis predicted 48,680 genes for pigeonpea and also showed the potential role of some gene families during evolution/domestication, e.g. drought tolerance-related genes. Although a few segmental duplication events were found, recent genome-wide duplication events, such as seen in soybean, were not observed. 


\subsubsection{Draft Genome Sequence from NRCPB (Singh et al. 2012)}

This draft genome, coordinated by NRCPB, was reported in Journal of Plant Biochemistry and Biotechnology. A number of research institutes, namely NRCPB, Division of Genetics, Indian Agricultural Research Institute, New Delhi; Institute of Agricultural Sciences, Banaras Hindu University, Varanasi; Indian Institute of Pulses Research, Kanpur; University of Agricultural Sciences, Dharwad; Panjabrao Deshmukh Krishi Vidyapeeth, Krishinagar, Akola from India participated in this consortium. This work was carried out with funding support from ICAR.

The consortium also used 'Asha' for assembling draft genome sequence data using long-sequence reads of 454 GS-FLX. A total of $0.51-\mathrm{Gb}$ high-quality sequence data were generated and analysed. In brief, sequence analysis provided a total of 47,004 protein-coding genes which is quite similar to Varshney et al. (2012). Further, 1213 disease resistance/defence response genes were detected.

In summary, both of above-mentioned sequencing efforts have made the first pulse crop with genome sequence information. These efforts have created a 'Supermarket' of genes and molecular markers. However, we understand that number of genes predicted in pigeonpea are inflated and may reduce with better quality of draft genome assembly in near future. It will lead to the identification and manipulation of candidate genes or genomic regions associated with resistance or tolerance to biotic and abiotic stresses, yield contributing and other agronomic traits to enable pure line or hybrid breeding.

\subsection{Deployment of Genome Sequence Information for Pigeonpea Improvement}

Availability of genomic resources, such as draft genome sequence alone is not enough to improve the crop productivity. In fact, the genome sequence is starting point and one of the important tools to harness genetic diversity for the traits of interest to crop improvement. In addition to developing the varieties or parental lines of hybrids with enhanced resistance to sterility mosaic disease (SMD) and fusarium wilt (FW), the pigeonpea breeding community requires early maturing as well as photo-period insensitive lines so that crop production can be expanded to new niches such as sloping hills and fit into the new production systems with short-time windows. While resistance to pod borers (Helicoveropa armigera and Maruca vitrata) is another interesting trait, limited or non-availability of resistance to these insects in the cultivated gene pool does not allow the use of a molecular breeding approach for targeting these traits (at least for now).

Although breeders have been engaged in developing superior varieties, the genetic base of pigeonpea is limited due to minimal usage of diverse genotypes in breeding programs, and there have been only a few breeding programs across the world. Also, modern breeding tools such as molecular breeding could not be deployed in breeding due to non-availability of marker-trait information for traits of interest. In this direction, development of draft genome sequence has been considered as a milestone in pigeonpea research. As a result of this breakthrough, a significant amount of information has become available. These resources can be used as tools to harness the genetic diversity for crop improvement.

Just after decoding of pigeonpea genome sequence (Nov. 7, 2011), round table discussions were organized on 15 November, 2011 by Mr P. Basu, the then Secretary and Mr Mukesh Khullar, the then Joint Secretary, Department of Agriculture \& Cooperation (DAC), Ministry of Agriculture, Government of India. In this meeting, representatives from different pigeonpea research institutes across India including the leaders of both genome sequencing projects-Dr Rajeev K. Varshney (IIPG/ICRISAT) and Dr NK Singh (NRCPB/ICAR) - and several other key stakeholders from India were present. In this important meeting, key issues related to pigeonpea improvement were identified. Subsequently, 
ICRISAT in collaboration with University of Agricultural Sciences, ARS - Gulbarga, Karnataka and the Professor Jayashankar Telangana State Agricultural University (PJTSAU)-Hyderabad, Telangana-developed a road map for deploying genome sequence information for pigeonpea improvement. The consortium brought expertise in different disciplines, namely breeding, genomics, pathology, and wide hybridization. These efforts led to the development of the project 'Pigeonpea improvement using molecular breeding' with an aim to harness diversity present in the breeding material and germplasm collection. Subsequently, the proposal was approved by Ministry of Agriculture, Government of India and United States Agency for International Development (USAID) - India Mission.

The project was planned in three phases, phase I (generating basic information/material to initiate molecular breeding), phase II (molecular breeding, multi-location evaluation, development and extension in Asia and Africa) and phase III (Coordinated Research Project trials and extension in Asia and Africa) (Fig. 8.1). Phase I (for three years) was initiated in the year 2012 with the funding from USAID-India. Furthermore, research efforts were expanded for deploying genome sequence information for pigeonpea improvement through support from Department of Biotechnology, Government of India; CGIAR Research Program on Grain Legumes.

Significant progress has been made in different areas including developing new genetic stocks, utilization of genetic material for genome-wide profiling through re-sequencing, genotyping by sequencing, SNP arrays and establishing marker-trait association. Some of this progress has been presented in the sections below.

\subsubsection{Developing New Genetic Stocks}

Pigeonpea has a very strong crop improvement programme; however, pedigree analysis of released cultivars indicates that $\mathrm{T}-1$ and $\mathrm{T}-190$ were the most frequently used parents (Kumar et al. 2004). There has been limited use of genetic diversity from germplasm collection in breeding programs in majority of crops including pigeonpea (Upadhyaya et al. 2011). Above-mentioned points clearly explain reasons behind the low level of genetic diversity observed in cultivated gene pool. Therefore, it was planned to develop new genetic stock with broader genetic base and deploy family-based mapping approaches such as MAGIC and NAM to tackle complex traits precisely. These family-based approaches that play an intermediate role between classical bi-parental and natural populations have been developed in some other crops such as maize and Arabidopsis (McMullen et al. 2009; Kover et al. 2009).

Multi-parent advanced generation intercross (MAGIC): MAGIC could be used for high-resolution mapping and generating new breeding material with combined features of various important traits. Thus, MAGIC directly enriches breeding efforts in two ways: first, by revealing best alleles underlying a given trait and second, by their enrichment into a single genotype that can be easily recognized. MAGIC populations have been developed in Arabidopsis (Kover et al. 2009), rice (Bandillo et al. 2009, 2013) and wheat (Huang et al. 2012). Therefore, development of MAGIC population in pigeonpea through reshuffling of the genome to enhance the genetic base and to identify the marker traits associations was initiated. A total of eight diverse founder parents (four elite breeding lines and four landraces) with desirable features were selected for the development of MAGIC population (Table 8.1). Half diallel crossing approach (28, two-way $\mathrm{F}_{1} \mathrm{~s}$ ) followed by funnel-based mating design (14, four-way and 7 , eight-way $F_{1} s$ ) is being utilized for the development of at least 1000 MAGIC lines.

Nested association mapping (NAM): The NAM approach involves several populations that have one common parent, with the other parental genotype contrasting for traits of interest to the common parent genotype. Using this approach, available genetic diversity in the elite cultivars/breeding lines is utilized (McMullen 


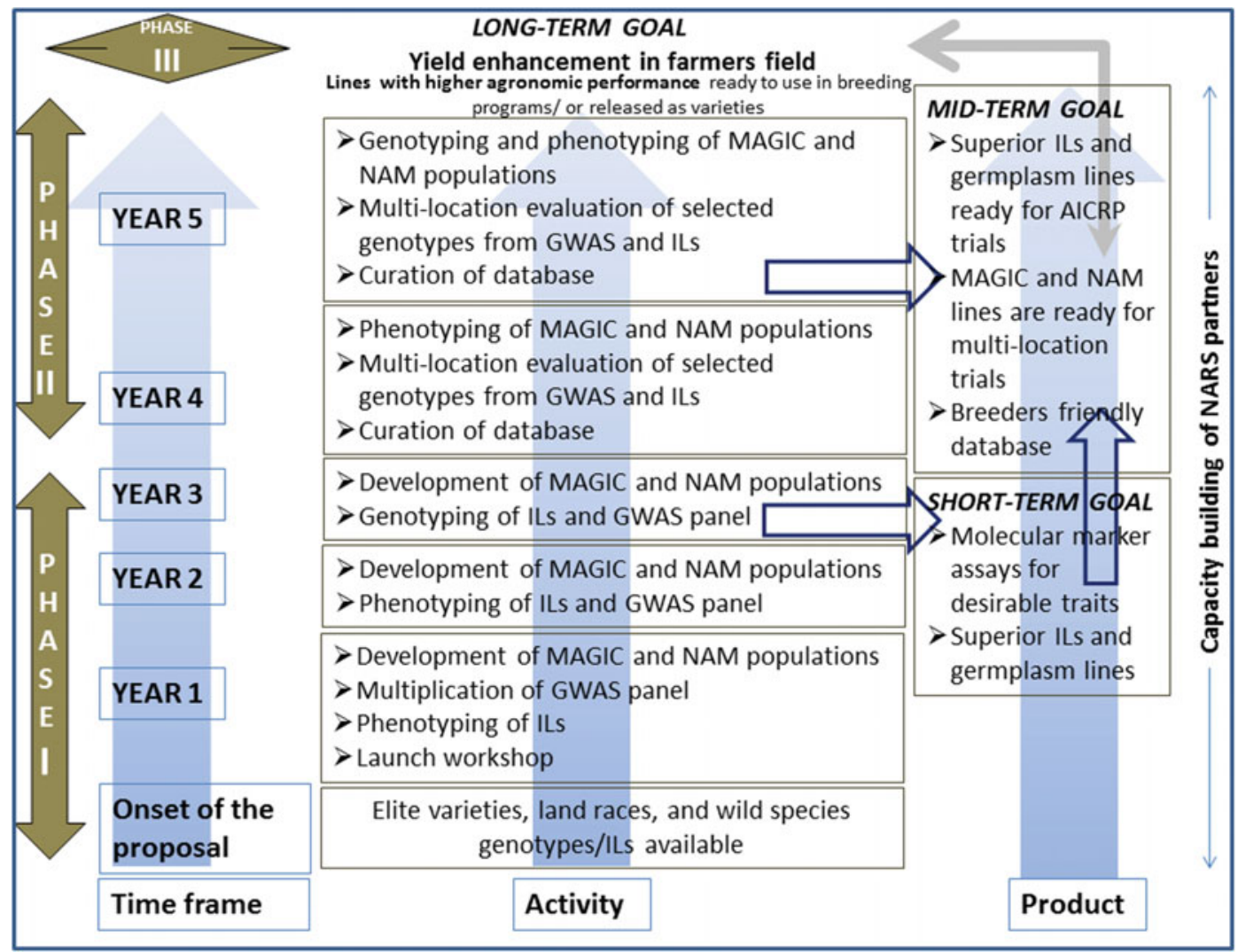

Fig. 8.1 A detailed plan for the deployment of genome sequence information for pigeonpea improvement in three phases, phase I (generating basic information/material to initiate molecular breeding), phase II (molecular breeding, multi-location evaluation, development and extension in Asia and Africa) and phase III (Coordinated Research Project trials and extension in Asia and Africa)

Table 8.1 List of parents and characteristic features used in the development of MAGIC population in pigeonpea

\begin{tabular}{l|l}
\hline Lines & Features \\
\hline ICP 7426 & High pod numbers, medium duration \\
\hline HPL 24 & $\begin{array}{l}\text { High protein content, medium duration, compact, susceptible to FW and resistant to SMD, } \\
\text { inter-specific derivative }\end{array}$ \\
\hline ICP 11605 & Early flowering, germplasm line \\
\hline ICP 14209 & High number of pods, germplasm line \\
\hline ICP 14486 & Early flowering, germplasm line \\
\hline ICP 5529 & Medium duration, obcordate leaves, compact plant, poor yielding, modified flower \\
\hline ICP 7035 & $\begin{array}{l}\text { Medium duration, SMD resistant to both Patancheru and Bangalore races, large purple seed, high } \\
\text { sugar content }\end{array}$ \\
\hline ICP 8863 & $\begin{array}{l}\text { Erect, mid-late, highly resistant to FW and susceptible to SMD, an extensively grown variety in } \\
\text { Northern Karnataka and Maharashtra region of India, red seeded genotype }\end{array}$ \\
\hline
\end{tabular}


Table 8.2 List of parents and characteristic features used in the development of NAM population

\begin{tabular}{|c|c|}
\hline Lines & Features \\
\hline \multicolumn{2}{|l|}{ Nested parent } \\
\hline Asha & Genome sequence available, leading variety, resistant to FW and SMD \\
\hline \multicolumn{2}{|c|}{ Founder parent } \\
\hline HPL 24 & $\begin{array}{l}\text { High protein content, medium duration, compact, susceptible to FW and resistant to SMD, } \\
\text { inter-specific derivative }\end{array}$ \\
\hline ICP 7035 & $\begin{array}{l}\text { Medium duration, SMD resistant to both Patancheru and Bangalore races, large purple seed, high } \\
\text { sugar content }\end{array}$ \\
\hline ICP 8863 & $\begin{array}{l}\text { Erect, mid-late, highly resistant to FW and susceptible to SMD, an extensively grown variety in } \\
\text { Northern Karnataka and Maharashtra region of India, red seeded genotype }\end{array}$ \\
\hline ICPL 87 & Early duration, determinate, short, high combiner \\
\hline ICPL 88039 & Extra early maturity, indeterminate, good yield \\
\hline ICPL 85063 & Medium duration, indeterminate, good yield, more branching \\
\hline MN 1 & Super early, small seeded, determinate \\
\hline ICP 28 & Early maturity, local varieties \\
\hline ICPL 85010 & Early maturity, local varieties \\
\hline ICP 7263 & Determinate, long podded, white seeded \\
\hline
\end{tabular}

et al. 2009). Progenies coming from the different sets of bi-parental populations can be analysed using both linkage as well as association mapping approach. Therefore, there is a need to develop NAM populations for targeted traits. In the case of pigeonpea, a total of 10 crosses involving Asha as a common parent have been made at ICRISAT (Table 8.2).

Developed MAGIC and NAM populations in near future will be analysed together with genome-wide markers and trait phenotyping data. Such populations have potential to not only provide the trait-associated markers, but also provide new genetic combinations for pigeonpea breeding program, which can be proved as a game changer in elevating productivity and genetic diversity.

\subsubsection{Utilizing Available Genetic Resources}

As described in previous chapters of this book on genomic resources and germplasm characterization, a number of mapping populations ranging from $\mathrm{F}_{2}$ s (Varshney et al. 2010b), recombinant inbred lines (RILs), advanced back-cross mapping populations and specialized germplasm collections, namely composite collection (1000 lines, Upadhyaya et al. 2011), core collection (1290 lines, Reddy et al. 2005), mini-core collection (146 lines, Upadhyaya et al. 2006) have been developed. There is another set of germplasm collection comprising of 300 genotypes called 'reference set'. This 'reference set' represents $95 \%$ of SSR alleles present in the composite collection and also a reservoir of several traits of interests to the breeders, e.g. early flowering, high number of pods, high 100-seed weights and high seed yield per plant. To map the target traits for biotic stress resistance (FW and SMD), three bi-parental mapping populations comprising of 188 lines in each were developed and designated as Pigeonpea Recombinant Inbred Line (PRIL) populations. At present, there are three populations, namely PRIL_A (ICPB $2049 \times$ ICPL 99050), PRIL_B (ICPL $20096 \times$ ICPL 332) and PRIL_C (ICPL $20097 \times$ ICPL 8863). Trait phenotyping of these mapping populations have been completed for two years at two to three locations. Based on the phenotypic datasets, FW and SMD resistance lines are being identified. 


\subsubsection{Whole-Genome Re-sequencing Initiatives}

Initial investments in developing draft genome sequence information in any organism provide dividend in the form of re-sequencing projects. Where genome-wide profiling of target sets in a given species becomes cost-effective. Especially, in the present world of low-cost NGS, an opportunity arises to catalogue genome-wide variations in a number of individuals. NGS together with draft genome sequence has enabled identification of SNPs and Indels in efficient and high-throughput manner in a number of species (Lam et al. 2010; Xu et al. 2012). In the case of pigeonpea, re-sequencing of different germplasm sets has been initiated. These sets include parents of mapping populations (Kumar et al. 2016), reference set (Varshney et al. 2017), parental lines of hybrids (unpublished). These datasets will subsequently enable the pigeonpea researchers to deploy GAB and to overcome the bottleneck of limited genetic diversity information.

First generation HapMap: The first report on whole-genome re-sequencing (WGRS) in pigeonpea has been published very recently (Kumar et al. 2016). In this effort, WGRS was conducted using Illumina paired-end sequencing technology on a panel of 20 Cajanus lines. Selected lines represent crossing parentals of RILs, introgression lines (ILs), MAGIC and NAM. Across the 20 lines, a total of $157 \mathrm{~Gb}$ raw data with an effective mapping depth of $\sim 12 \mathrm{X}$ per genotype were generated. Subsequently, a total of 5.4 million variations including 4.6 million SNPs and 0.7 million Indels were identified across the lines. This study also provided 2598 copy number variations (CNVs) and 970 presence and absence variations (PAVs). Additionally, unique accession/genotype signatures were also detected through genome-wide analysis. The analysis clearly explained a narrow genetic base in cultivated gene pool and suggested use of new populations such as IL or $\mathrm{AB}$ populations, MAGIC, NAM to re-introduce adaptive diversity in pigeonpea breeding.

Reference set: To catalogue the sequence diversity, we have re-sequenced the genomes of
292 lines from a total of 300 pigeonpea lines in reference set on HiSeq 2500 platform. This set is comprised of breeding lines, landraces and wild species lines. WGRS yielded $2.15 \mathrm{~Tb}$ of sequence data with the coverage ranging from 5 to $12 \mathrm{X}$. The WGRS data have been used to identify small variations (SNPs and Indels) and large structural variations (CNVs and PAVs). Re-sequencing data have provided variation counts around 17 million across 292 Cajanus lines (Varshney et al. 2017). This generated sequence data have been used to understand genetic relationships among Cajanus lines, targets of domestication and human selection genetic sweeps and associations between genomic regions with agronomic important traits.

Parental lines of hybrids: In order to define heterotic pools in pigeonpea, a set of 104 parental lines (cytoplasmic male sterile, maintainers and restorers) have been re-sequenced. WGRS yielded 511 GB sequence data with the coverage ranging from 5 to $10 \mathrm{X}$. A total of 3.4 million SNPs could be identified across 104 lines (unpublished). In parallel, it has been planned to develop testcrosses from the parental lines. $F_{1}$ hybrids along with parental lines will be phenotyped for yield-related traits. The combined analysis with genome-wide variations and phenotypic data would provide clues on hybrid performance that will be helpful in accelerating hybrid improvement.

\subsubsection{Sequencing-Based Genotyping Approaches}

Pigeonpea draft genome sequence has provided opportunities to deploy sequencing-based genotyping approaches such as GBS and QTL-seq to generate high-density linkage maps and marker identification. GBS provides ease of discovery and genotyping of markers in a single-step process. GBS technique is now being used for genotyping PRILs (_A,_B and_C), multiple $\mathrm{F}_{2}$ populations and ILs for developing high-density pigeonpea genetic maps and subsequently for identification of traits-associated markers (Saxena et al. 2017a, b). Another approach of marker 
detection combines bulked segregant analysis (BSA) and WGRS has been deployed in pigeonpea for identification of genomic region(s) responsible for FW and SMD resistance (Singh et al. 2016). In brief, R-bulk (resistant bulk) and S-bulk (susceptible bulk) along with the resistant parent were re-sequenced for identification of candidate genomic region(s)/genes. Detailed analysis of sequence data identified association of four candidate nsSNPs in four genes with FW resistance and four candidate nsSNPs in three genes with SMD resistance (for detailed results please see Singh et al. 2016).

\subsubsection{High-Density SNP Array (Axiom ${ }^{\circledR}$ CajanusSNP Array)}

In order to use above-mentioned sequence variations identified through large-scale WGRS in pigeonpea, an array targeting $\sim 56,000$ SNPs has been developed using the Affymetrix platform. SNP probes were designed by screening $\sim 2$ million SNP loci extracted from the re-sequencing data of cultivated lines. A total of 56,512 SNPs were placed on array. The array contained evenly distributed markers in the genome. Initial genotyping showed that this array had high genotyping accuracy and could be used for different objectives (unpublished). Therefore, it has been planned to genotype pigeonpea composite collection ( $\sim 1000$ lines) and at least three different mapping populations segregating for important traits with above-mentioned 'Axiom ${ }^{\circledR}$ CajanusSNP Array'. This array will play an important role in genomics studies and molecular breeding.

\subsection{Conclusion}

Two draft genome assemblies have been generated in pigeonpea by collaborative sequencing initiatives. These draft genomes have provided information on genes and subsequently led the foundation of a number of re-sequencing projects and applications of novel methodologies. The information produced through re-sequencing projects is continuously adding up and will be surely helpful in understanding the crop evolution, domestication, genetic relationships, genetic control of genes, biological process in different metabolic pathways etc. Whereas novel approaches such as GBS and SNP arrays together with trait phenotyping data will provide candidate markers/genes associated with different important traits in pigeonpea. As a next step in pigeonpea genomics, there is a need of improvement in draft genome assembly where PACBio reads along with improved analysis algorithms will certainly help. It is anticipated that coming years will be witnessing usage of genome sequence information in developing superior lines of pigeonpea for both varietal and hybrid breeding.

Acknowledgements Authors are thankful to the United States Agency for International Development (USAID), Biotechnology Industry Partnership Programme (BIPP) and Department of Biotechnology of Government of India for supporting various activities related to pigeonpea genomics and molecular breeding projects at ICRISAT. This work has been undertaken as part of the CGIAR Research Program on Grain Legumes. ICRISAT is a member of CGIAR Consortium.

\section{References}

Bandillo N, Muyco PA, Caspillo C, Laza M, Sajise AG, Redoña E, Singh RK, Leung H (2009) Population development through multiparent advanced generation intercrosses (MAGIC) among diverse genotypes to facilitate gene discovery for various traits in rice. Philippine J Crop Sci 34

Bandillo N, Raghavan C, Muyco PA, Sevilla ALM, Lobina TI, Dilla-Ermita CJ, Wei C, Tung CW, McCouch S, Thomson M, Mauleon R, Singh RK, Gregorio G, Redoña E, Leung H (2013) Multi-parent advanced generation inter-cross (MAGIC) populations in rice: progress and potential for genetics research and breeding. Rice 6:1-15

Bohra A, Saxena RK, Gnanesh BN, Saxena KB, Byregowda M, Rathore A, KaviKishor PB, Cook DR, Varshney RK (2012) An intra-specific consensus genetic map of pigeonpea [Cajanus cajan (L.) Millspaugh] derived from six mapping populations. Theor Appl Genet 125:1325-1338

Gnanesh BN, Bohra A, Sharma M, Byregowda M, Pande S, Wesley V, Saxena RK, Saxena KB, KaviKishor PB, Varshney RK (2011) Genetic mapping and quantitative trait locus analysis of resistance 
to sterility mosaic disease in pigeonpea [Cajanus cajan (L.) Millsp.]. Field Crops Res 123:53-61

Huang BE, George WA, Forrest KL, Kilian A, Hayden JM, Morell KM, Cavanagh RC (2012) A multiparent advanced generation inter-cross population for genetic analysis in wheat. Plant Biotechnol J 10:826839

Kover PX, Valdar W, Trakalo J, Scarcelli N, Ehrenreich IM, Purugganan MD et al (2009) A multiparent advanced generation inter-cross to fine-map quantitative traits in Arabidopsis thaliana. PLoS Genet 5: e1000551. doi:10.1371/journal.pgen.1000551

Kumar S, Gupta S, Chandra S, Singh BB (2004) How wide is genetic base of pulse crops? In: Ali M, Singh BB, Kumar Shiv and Vishwa Dhar (eds) Pulses in new perspective. Proceedings of the national symposium on crop diversification and natural resources management, 20-22 December 2003. Indian Society of Pulses Research and Development, Indian Institute of Pulses Research, Kanpur, pp 211-221

Kumar V, Khan AW, Saxena RK, Garg V, Varshney RK (2016) First generation hapmap in Cajanus spp. reveals untapped variations in parental lines of mapping populations. Plant Biotechnol J 14:16731681. doi:10.1111/pbi. 12528

Kumawat G, Raje RS, Bhutani S, Pal JK, Mithra AS, Gaikwad K, Sharma TR, Singh NK (2012) Molecular mapping of QTLs for plant type and earliness traits in pigeonpea (Cajanus cajan L. Millsp.). BMC Genet 13:1

Lam HM, Xu X, Liu X, Chen W, Yang G, Wong FL, Li MW, He W, Qin N, Wang B, Li J, Jian M, Wang J, Shao G, Wang J, Sun SSM, Zhang G (2010) Resequencing of 31 wild and cultivated soybean genomes identifies patterns of genetic diversity and selection. Nat Genet 42:1053-1059

McMullen MD, Kresovich S, Villeda HS, Bradbury P, Li H et al (2009) Genetic properties of the maize nested association mapping population. Science 325:737-740

Reddy LJ, Upadhyaya HD, Gowda CLL, Reddy KN, Singh S (2005) Development of core collection in pigeon pea [Cajanus cajan (L) Millspaugh] using geographic and qualitative morphological descriptors. Genet Resour Crop Evol 52:1049-1056

Saxena RK, Singh VK, Kale SM, Tathineni R, Parupalli S, Kumar V, Garg V, Das RR, Sharma M, Yamini KN, Muniswamy S, Ghanta A, Rathore A, Sameer Kumar CV, Saxena KB, Kavi Kishor PB, Varshney RK (2017a) Construction of genotypingby-sequencing based high-density genetic maps and QTL mapping for fusarium wilt resistance in pigeonpea. Sci Rep 7:1911

Saxena RK, Kale SM., Kumar V, Parupalli S, Joshi S, Singh VK, Garg V, Das RR, Sharma M, Yamini KN, Ghanta A, Rathore A, Sameer Kumar CV, Saxena KB, Varshney RK (2017b) Genotyping-by-sequencing of three mapping populations for identification of candidate genomic regions for resistance to sterility mosaic disease in pigeonpea. Sci Rep 7:1813

Singh NK, Gupta DK, Jayaswal PK, Mahato AK, Dutta S et al (2012) The first draft of the pigeonpea genome sequence. J Plant Biochem Biotechnol 21:98. doi:10. 1007/s13562-011-0088-8

Singh VK, Khan AW, Saxena RK, Kumar V, Kale SM, Sinha P, Chitikineni A, Pazhamala LT, Garg V, Sharma M, Sameerkumar CV, Parupalli S, Suryanarayana V, Patil S, Muniswamy S, Ghanta A, Yamini KN, Dharmaraj PS, Varshney RK (2016) Next generation sequencing for identification of candidate genes for fusarium wilt and sterility mosaic disease in pigeonpea (Cajanus cajan). Plant Biotechnol J 14:1183-1194. doi:10.1111/pbi.12470

Upadhyaya HD, Gowda CLL, Buhariwalla HK, Crouch JH (2006) Efficient use of crop germplasm resources: identifying useful germplasm for crop improvement through core and min-core collections and molecular marker approaches. Plant Genet Resour 4:25-35

Upadhyaya HD, Reddy KN, Sharma S, Varshney RK, Bhattacharjee R, Singh S, Gowda CLL (2011) Pigeonpea composite collection and identification of germplasm for use in crop improvement programmes. Plant Genet Resour 9:97-108

Varshney RK, Graner A, Sorrells ME (2005) Genomics assisted breeding for crop improvement. Trends Plant Sci 10:621-630

Varshney RK, Hoisington DA, Tyagi AK (2006) Advances in cereal genomics and applications in crop breeding. Trends Biotechnol 24:490-499

Varshney RK, Hoisington DA, Nayak SN, Graner A (2010a) Molecular plant breeding: methodology and achievements. In: Somers D, Langridge P, Gustafson PJ (eds) Methods in molecular biology: plant genomics. The Humana Press, TotowaNJ, USA, pp 283-304

Varshney RK, Penmetsa RV, Dutta S, Kulwal PL, Saxena RK, Datta S et al (2010b) Pigeonpea genomics initiative (PGI): an international effort to improve crop productivity of pigeonpea (Cajanus cajan L.). Mol Breed 26:393-408

Varshney RK, Chen W, Li Y, Bharti AK, Saxena RK, Schlueter JA et al (2012) Draft genome sequence of pigeonpea (Cajanus cajan), an orphan legume crop of resource-poor farmers. Nat Biotechnol 30:83-89

Varshney RK, Gaur PM, Chamarthi SK, Krishnamurthy L, Tripathi S, Kashiwagi J, Samineni S, Singh VK, Thudi M, Jaganathan D (2013) Fast-track introgression of "QTL-hotspot" for root traits and other drought tolerance traits in JG 11, an elite and leading variety of chickpea. The Plant Genome. doi:10.3835/ plantgenome2013.07.0022

Varshney RK, Pandey MK, Janila P, Nigam SN, Sudini H, Gowda MVC, Sriswathi M, Radhakrishnan T, Manohar SS, Nagesh P (2014) Marker-assisted introgression of a QTL region to improve rust 
resistance in three elite and popular varieties of peanut (Arachis hypogaea L.). Theor Appl Genet 127:17711781

Varshney RK, Saxena RK, Upadhyaya HD, Khan AW, Yu Y, Kim C, Rathore A, Kim D, Kim J, An S, Kumar V, Anuradha G, Yamini KN, Zhang W, Muniswamy S, Kim J-S, Penmetsa RV, Wettberg EV, Datta SK (2017) Whole-genome resequencing of 292 pigeonpea accessions identifies genomic regions associated with domestication and agronomic traits. Nat Genet 49:1082-1088

Xu X, Liu X, Ge S, Jensen JD, Hu FY, Li X, Dong Y, Gutenkunst RN, Fang L, Huang L, Li JX, He WM, Zhang GJ, Zheng XM, Zhang FM, Li YR, Yu C, Kristiansen K, Zhang XQ, Wang J, Wright M, McCouch S, Nielsen R, Wang J, Wang W (2012) Resequencing 50 accessions of cultivated and wild rice yields markers for identifying agronomically important genes. Nat Biotechnol 3:105-157 